\title{
Collocating satellite-based radar and radiometer measurements - methodology and usage examples
}

\author{
G. Holl ${ }^{1}$, S. A. Buehler ${ }^{1}$, B. Rydberg ${ }^{2}$, and C. Jiménez ${ }^{3}$ \\ ${ }^{1}$ Department of Space Science, Luleå University of Technology, Kiruna, Sweden \\ ${ }^{2}$ Department of Radio and Space Science, Chalmers University of Technology, Göteborg, Sweden \\ ${ }^{3}$ Laboratoire d'Etude du Rayonnement et de la Matière en Astrophysique, Centre National de la Recherche Scientifique, \\ Observatoire de Paris, Paris, France
}

Received: 29 January 2010 - Published in Atmos. Meas. Tech. Discuss.: 26 February 2010

Revised: 20 May 2010 - Accepted: 1 June 2010 - Published: 21 June 2010

\begin{abstract}
Collocations between two satellite sensors are occasions where both sensors observe the same place at roughly the same time. We study collocations between the Microwave Humidity Sounder (MHS) on-board NOAA-18 and the Cloud Profiling Radar (CPR) on-board CloudSat. First, a simple method is presented to obtain those collocations and this method is compared with a more complicated approach found in literature. We present the statistical properties of the collocations, with particular attention to the effects of the differences in footprint size. For 2007, we find approximately two and a half million MHS measurements with CPR pixels close to their centrepoints. Most of those collocations contain at least ten CloudSat pixels and image relatively homogeneous scenes. In the second part, we present three possible applications for the collocations. Firstly, we use the collocations to validate an operational Ice Water Path (IWP) product from MHS measurements, produced by the National Environment Satellite, Data and Information System (NESDIS) in the Microwave Surface and Precipitation Products System (MSPPS). IWP values from the CloudSat CPR are found to be significantly larger than those from the MSPPS. Secondly, we compare the relation between IWP and MHS channel 5 $(190.311 \mathrm{GHz})$ brightness temperature for two datasets: the collocated dataset, and an artificial dataset. We find a larger variability in the collocated dataset. Finally, we use the collocations to train an Artificial Neural Network and describe how we can use it to develop a new MHS-based IWP product. We also study the effect of adding measurements from the High Resolution Infrared Radiation Sounder (HIRS), channels $8(11.11 \mu \mathrm{m})$ and $11(8.33 \mu \mathrm{m})$. This shows a small improvement in the retrieval quality. The collocations described in the article are available for public use.
\end{abstract}

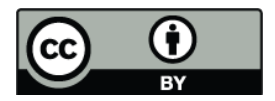

Correspondence to: G. Holl (gerrit.holl@ltu.se)

\section{Introduction}

Atmospheric remote sensing from satellites is a major source of data for the atmospheric sciences and for operational weather forecasting (Kidd et al., 2009). Measurements from Earth observation satellites have a global or near-global coverage. However, the accuracy of products derived from such measurements is often poor (Wielicki et al., 1995; Wu et al., 2009). A combination of observations from different instruments enables applications that are impossible with singleinstrument measurements. One way to combine measurements is through collocations. A collocation is an event where different (satellite) sensors observe the same location at roughly the same time. The collocations considered here are mainly between active measurements from the Cloud Profiling Radar on-board CloudSat, and passive measurements from microwave and infrared sensors on-board $\mathrm{Na}-$ tional Oceanic and Atmospheric Administration (NOAA)18.

One product obtained by remote sensing measurements is the Ice Water Path (IWP), the vertically integrated Ice Water Content (IWC) or the column mass density of ice in the atmosphere. Ice clouds are important for the climate, because they absorb and scatter thermal radiation and reflect solar radiation, and thus influence the radiation budget of the Earth (Stephens, 2005). As shown by John and Soden (2006), different General Circulation Models (GCMs) disagree by an order of magnitude about the climatology of IWP. Also IWP values from remote sensing measurements differ considerably (Wu et al., 2009). Therefore, it is important to improve the quality of ice cloud retrievals. A good understanding of the cloud signal in microwave radiometer measurements is an important step in the development of retrieval algorithms for possible future missions, such as the Cloud Ice Water Submillimetre Imaging Radiometer (CIWSIR), proposed by Buehler et al. (2007).

Published by Copernicus Publications on behalf of the European Geosciences Union. 
Collocations between sensors on the same platform are commonly used (for example, see Frey et al., 1996; Bennartz, 2000). The idea to collocate data from different satellite platforms is not new either. Wielicki and Parker (1992) compare the cloud cover obtained with sensors of different spatial resolution. The A-Train constellation was motivated by the advantages of using a combination of measurements (Stephens et al., 2002). Already before CloudSats launch, Miller et al. (2000) described how to use active sensor data as a priori information for passive sensor retrievals, anticipating "a considerable overlap of CloudSat with the Earth Observing System (EOS) PM and Geostationary Operational Environmental (GOES) satellites". Several recent studies use the new possibilities from the A-Train (for example, Holz et al., 2008; Kahn et al., 2008). However, not much work has been published on actual collocation methods. The first publication on the subject appears to be a technical note written in Japanese (Aoki, 1980). Judging from the abstract, Aoki (1980) describes how to match AVHRR and HIRS/2 if the instruments are on the same satellite. Other conference papers on the subject are Nagle (1998) and Sun et al. (2006). The first peerreviewed publication on the subject appears to be Nagle and Holz (2009), discussed in more detail in Sect. 3.1.1.

No literature exists that focusses on collocations between an active instrument such as the Cloud Profiling Radar (CPR) on-board CloudSat and passive, operational instruments on Polar Orbiting Environmental Satellites (POES) such as the MHS on the NOAA-18. However, such collocations have relevant applications. Although a satellite like CloudSat has high quality products, the coverage is small compared to operational satellites, and it will have a limited lifetime. If we can use collocations between CloudSat CPR and NOAA-18 MHS to improve the operational microwave IWP retrieval, the advantages will last much beyond the lifetime of the ATrain satellites and have a much higher spatial coverage. Even passive microwave data from before CloudSat could be reprocessed with an improved algorithm. Whereas Miller et al. (2000) describe a retrieval that requires collocated data for each individual retrieval, we show that collocations can be used to develop new retrievals, that can then be used for non-collocated passive radiometer measurements.

The main purpose here is to study collocations between CloudSat CPR and NOAA-18 MHS. Collocations with MHS and AMSU-B on other POES were also located, but due to the large distances between the satellites, few useful collocations were found. Hence, the study focuses on NOAA18 MHS. The collocation procedure is described in Sect. 3. The secondary purpose of the study is to look at possible uses of the collocations. Three applications are described in Sect. 4. Firstly, the NOAA National Environmental Satellite, Data and Information Service (NESDIS) Microwave Surface and Precipitation Products System (MSPPS) IWP product is compared with the IWP product from the CPR on-board CloudSat (Sect. 4.1). Simulated radiances from generated clouds are used to study the relation between brightness tem- perature and IWP, and compare this with the statistics of the collocated dataset (Sect. 4.2). Finally, in Sect. 4.3, we use microwave radiances, with and without infrared measurements, to train an Artificial Neural Network with the CloudSat IWP as a target. Such a network can then be used to develop a new IWP product from microwave (and IR) measurements. Such applications were not found in peer-reviewed literature.

\section{Instruments}

The Cloud Profiling Radar (CPR) is a radar instrument on-board the sun-synchronous CloudSat satellite (Stephens et al., 2002), launched 28 April 2006. It has an operating frequency of $94 \mathrm{GHz}$ and measures profiles of backscattering ratio at a $0.16^{\circ}$ off-nadir angle. CloudSat generates a profile every $1.1 \mathrm{~km}$ along-track. A profile footprint is $1.3 \mathrm{~km}$ across-track and $1.7 \mathrm{~km}$ along-track. A profile is taken every $0.16 \mathrm{~s}$. CloudSat is part of the A-Train constellation. It has an inclination of $98.26^{\circ}$ and a Local Time Ascending Node (LTAN) varying between 13:30 and 13:45 local solar time. We use the ROIWP (Radar-Only Ice Water Path) field from the $2 B-C W C-R O$ (level 2b, Cloud Water Content, Radar Only) product, version 008. Austin et al. (2009) describe the algorithm to calculate IWC from radar reflectivity profiles. They report an upper limit of the uncertainty of $40 \%$. However, throughout this article, we assume CloudSat CPR to represent the truth since it is supposed to provide the most accurate measurements of IWP. The data originate from the CloudSat Data Processing Center. All measurements are geolocated and time-associated.

The Advanced Microwave Sounding Unit-B (AMSU-B) and its successor the Microwave Humidity Sounder (MHS) are microwave radiometers (Saunders et al., 1995; Kleespies and Watts, 2007). MHS channels 3-5 correspond to AMSUB channels 18-20. We use the MHS channel numbers. Channel 3 has a centre frequency of $183.31 \pm 1.00 \mathrm{GHz}$ with a bandwidth of $500 \mathrm{MHz}$, channel 4 has a centre frequency of $183.31 \pm 3.00 \mathrm{GHz}$ with a bandwidth of $1000 \mathrm{MHz}$, and channel 5 has a centre frequency of $183.31 \pm 7.00 \mathrm{GHz}$ (AMSUB) or $190.31 \mathrm{GHz}$ (MHS) with a bandwidth of $2000 \mathrm{MHz}$ (AMSU-B) or $2200 \mathrm{MHz}$ (MHS). We use channels 3-5 because of the prominent water vapour spectral line at 183.31 GHz. In this article, we neglect the differences between AMSU-B and MHS. Although they are not the same, the standard deviation of the difference is much larger than the mean difference, so that a simple correction is not possible (Kleespies and Watts, 2007). Because of its proximity to CloudSat, we focus on NOAA-18 and MHS for the collocations. However, we have also looked for collocations with MetOp-A (a satellite operated by the European Organisation for the Exploitation of Meteorological Satellites (EUMETSAT)), NOAA-15, NOAA-16 and NOAA-17, so with a total of five satellites. The MHS field of view is 
around $1.1^{\circ}$, and the footprint size at nadir is around $15 \mathrm{~km}$ in diameter. It scans across-track in angles from $-49.44^{\circ}$ to $49.44^{\circ}$ with 90 measurements per scan line. A scan takes $8 / 3 \mathrm{~s}$. MHS is currently present on NOAA-18, NOAA-19 and MetOp-A, whereas AMSU-B is present on NOAA-15 through NOAA-17. All those satellites are sun-synchronous satellites. NOAA-18 has an inclination of $98.74^{\circ}$ and a LTAN of $13: 39^{1}$. This is close to CloudSat, which leads to a large number of collocations, as described later in the article.

MHS measures the antenna temperature, which can be calibrated to obtain a brightness temperature in units of Kelvin. We use the ATOVS and AVHRR Pre-processing Package (AAPP) software package to apply this calibration, described by Labrot et al. (2006) (ATOVS stands for Advanced TIROS Operational Vertical Sounder, where TIROS stands for Television InfraRed Observation Satellite). We obtain the radiances from the NOAA CLASS archive.

All those satellites also carry the infrared radiometer High Resolution Infrared Radiation Sounder (HIRS), either HIRS/3 or HIRS/4. HIRS measures in 20 channels, one visible and nineteen infrared. We use channels $8(\lambda=11.1 \mu \mathrm{m}$, a window channel) and $11(\lambda=7.33 \mu \mathrm{m}$, a humidity channel) because ice clouds are clearly visible at those wavelengths. HIRS/3 is present on NOAA-15 through NOAA-17 and HIRS/4 is present on NOAA-18, NOAA-19 and MetOpA. HIRS scans the atmosphere in 56 angles between $-49.5^{\circ}$ and $49.5^{\circ}$. Those measurements are not on the same grid as the MHS measurements (see Fig. 1). A HIRS scan takes $6.4 \mathrm{~s}$.

\section{Finding collocations}

The footprint size of the considered sensors is in the order of kilometres, whereas the measurement duration is in the order of milliseconds. The spatial extent of a measurement is of the same order as the physical extent of a cloud or larger (kilometers), but the time order of a measurement (fraction of a second) is much smaller than a typical cloud lifetime (minutes to hours) (Rogers and Yau, 1979).

Thus, to have a meaningful collocation, the footprints need to have a physical overlap. However, the time between the measurements can be much larger than the duration of a measurement. Hence, a collocation occurs when the sensors observe exactly the same place at approximately the same time.

As shown in Fig. 1, an MHS footprint is an order of magnitude larger than a CPR footprint and HIRS measurements are not on the same grid as MHS measurements.

We create two collocated datasets. In the first dataset, there is an entry for each CloudSat measurement collocating with an MHS measurement, so that there can be many collocations for the same MHS pixel. In the second dataset, each collocation has a unique MHS measurement and CPR pixels are

\footnotetext{
${ }^{1}$ As of 5 February 2009 00:00:00 from the Polar Orbiting Environmental Satellites (POES) Spacecraft Status website.
}

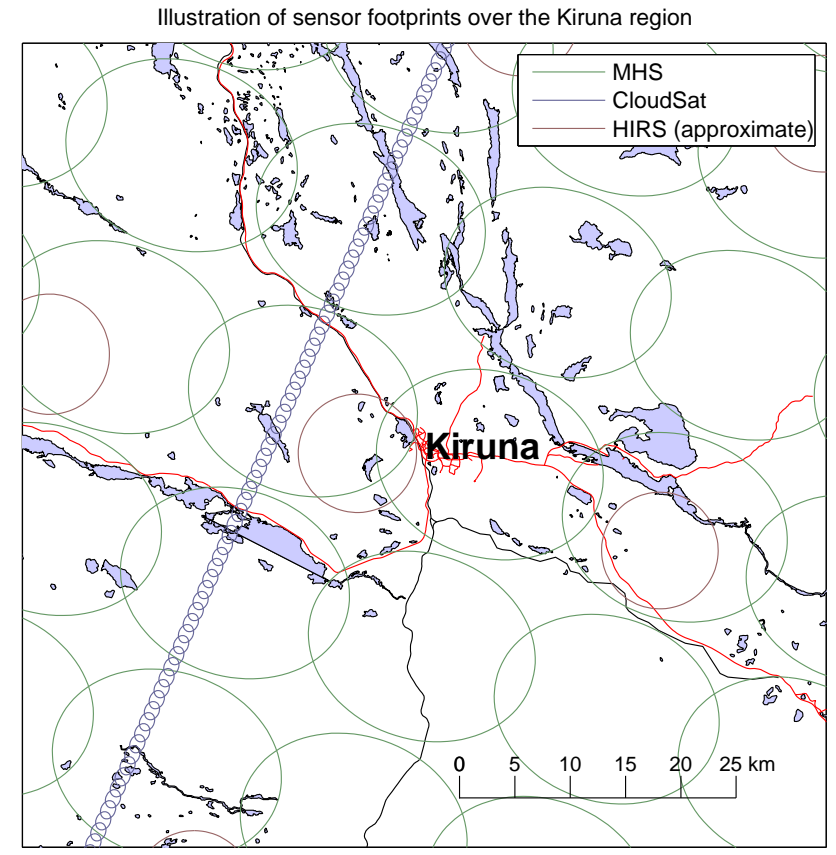

Fig. 1. Footprint of the MHS, HIRS/4 and CPR sensors. The MHS footprint sizes are calculated using an expression by Bennartz (2000). The HIRS footprints are approximate. Map data COOpenStreetMap contributors, CC-BY-SA.

averaged. For each MHS measurement, we note the number of CPR pixels inside the MHS pixel, the average CPR IWP value, the standard deviation of the CPR IWP and the fraction of cloudy CPR pixels. For the averaging, we consider the CPR pixels as point measurements and the MHS pixels as circular measurements with a radius of $7.5 \mathrm{~km}$ and a constant sensor spatial response function inside this area. In reality, the sensor spatial response function of MHS is better approximated by a Gaussian shape. Although this might reduce the representativeness, this effect is small compared to other error sources. The total area covered by the CPR pixels is still much smaller than the MHS footprint area. This leads to a sampling error, as discussed in Sect. 3.3 below.

Both datasets are available for public use.

\subsection{Collocation finding procedure}

The collocation finding procedure consists of four steps. The steps are described in detail in the following text.

1. Orbits (granules) with time overlap are selected.

2. Orbit sections are selected according to a rough temporal criterion.

3. Measurements possibly fulfilling the spatial criterion are selected. 
4. The temporal criterion is applied to the selected measurements.

The measurement data as obtained from the data providers is stored as one file for each orbit. Those files, known as granules, contain geolocated, time-referenced measurements. The geolocation refers to the actual measurement; the position of the satellite is not available and not required for the procedure (in contrast to Nagle and Holz (2009) discussed further down). The filenames contain information about the starting and ending time of the data contained by the granule.

For each CPR granule, we locate all NOAA and MetOp granules that have a time overlap with the CPR granule. Those are two granules for each POES for each CPR granule, or a total of ten files for each CPR granule to search for collocations (MetOp-A and NOAA-15 through NOAA-18).

We read the CPR file along with each of the associated POES files. The start and end times of the files are different. The segment with time overlap is selected, plus the segment where the time difference is less than the maximum time interval for a collocation to be considered. For example, if the CPR granule covers 10:00-11:30 UT, and a POES granule covers 11:00-12:30 UT, and our maximum time difference is $15 \mathrm{~min}$, we consider the data in the interval 10:45-11:45, or more precisely 10:45-11:30 in the CPR granule and 11:0011:45 in the POES granule.

As defined above, a collocation has a spatial and a temporal criterion. We use a two-step approach: first we look for any collocations that might meet the spatial criterion, and then whether those also meet the time criterion.

Starting from the orbit data screened according to the first temporal criterion as explained above, we find the measurements that meet the spatial criterion. In the first step, we do not consider the true pixel size or the sensor spatial response function of either sensor. Instead, we treat the measurements as points and define a maximum distance to select the measurement pair for further consideration. The sensor spatial response function and the effective field of view can be used later to select a subset of those collocations or a weighting of them to consider the MHS spatial response function.

We consider the ground track of each scan angle of the MHS (track $A$ ) and compare it to the single scan in the CPR (track $B$ ), but the following procedure works as well if both instruments are scanning.

If two ground tracks are plotted, a human observer can see immediately whether there is any spatial overlap or not. Computers can not, so the following algorithm is used to identify points where the spatial overlap condition is met.

1. The distance in $\mathrm{km}$ between successive points of the ground track is computed for both ground tracks, considering only the segments screened according to the temporal criterion discussed above. The maximum speed of the ground tracks is assumed to be the maximum distance.

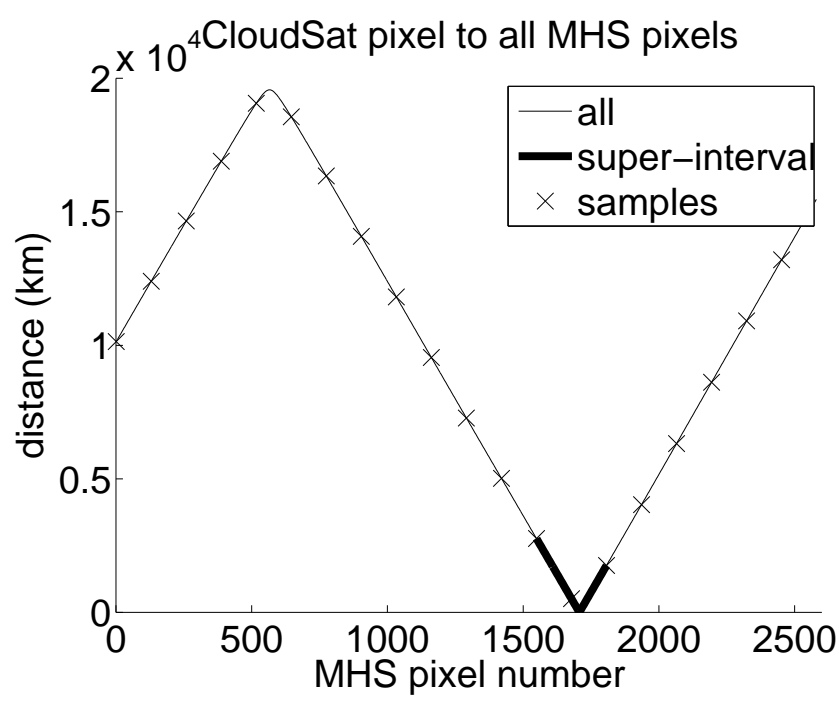

Fig. 2. Illustration of collocation principle. For this example, we consider the CloudSat granule starting 6 January 2007 at 01:10 UTC and the MHS granule starting at the same date at 00:26 UTC. The figure shows the distance from pixel number 11166 from the CloudSat granule to all MHS pixels at a viewing angle of $-0.56^{\circ}$. The crosses show twenty equally spaced samples and the thick line shows the super-interval to be searched for collocations. Refer to the text for further explanation.

2. Start with $n=1$.

3. Find close points to $A_{n}$ in $B$. Here, $A_{n}$ is the $n$-th measurement in track $A$. Figure 2 shows the distance from a CloudSat CPR pixel to all pixels in a MHS track for a fixed viewing angle. If any collocations exist, they will be close to the global minimum. Find points meeting the distance criterion by the following method.

(a) Choose $N$ equidistant points (henceforth samples) from $B$ as shown in Fig. 2. Combined with the first and the last point of the track, the samples define the edges for $N+1$ intervals. All intervals contain the same number of points, with the exception of the last interval, that may contain less points than the others.

(b) Find which sample is closest to $A_{n}$. Call this sample $B_{m}$.

(c) Consider $B_{m+1}, B_{m+2}, \cdots, B_{m+r}$ where $B_{m+r}$ is the first sample that does not meet the spatial condition or the last measurement point of the granule. Consider $B_{m-1}, B_{m-2}, \cdots, B_{m-l}$ where $B_{m-l}$ is the first sample that does not meet the spatial condition or the first point of the granule. If $N$ is large enough, all points that meet the spatial criterion are contained by the super-interval $\left(B_{m-l}, B_{m+r}\right)$, because the minimum of the distance from $A_{n}$ to $B$ will be contained by it (if $N$ is too small, this interval 
may contain only a local minimum). An example of such a super-interval is shown by the thick line in Fig. 2. Consider this super-interval.

(d) Calculate the distance between $A_{n}$ and every point in the super-interval.

(e) Note all points for which the spatial condition is met. If there are no such points, remember the distance of the closest point.

As shown in Fig. 2, $N=20$ is already sufficiently large to guarantee that any points in $B$ meeting the spatial criterion are contained in the super-interval. However, with $N=20$ the number of points in the super-interval for which the distance to $A_{n}$ will be calculated is still quite large. A larger $N$ means the super-interval will be smaller, but the number of samples for which the distance will be calculated will be larger. The choice of $N$ is thus an optimisation problem to reduce the number of distance calculations. We have chosen $N=200$.

4. If there were any points for with the spatial condition was met, increase $n$ by 1 and start again from 3 .

5. If there were no points for which the spatial condition was met, calculate the least number of points remaining before it could be met: increase $n$ by

$\underline{\text { smallest_distance - spatial_condition }}$ max_speed

and start again from 3. For example, if the shortest distance is $120 \mathrm{~km}$, the spatial condition distance $20 \mathrm{~km}$, and the max speed $10 \mathrm{~km} /$ point, $n$ will be increased by $\frac{120-20}{10}=10$.

This works, because if the minimum distance from $A_{n}$ to $B$ is $120 \mathrm{~km}$ and the distance between $A_{n}$ and $A_{n+10}$ is $100 \mathrm{~km}$, the maximum distance between $A_{n+10}$ and $B$ cannot be less than $20 \mathrm{~km}$.

The procedure described above is not the fastest possible (for example, point (d) could be optimised further) but with this algorithm, the bulk of the time running the code searching for collocations was spent on downloading files from a local server and decompressing them.

From all points obtained with the procedure described above, those for which the time difference is less than $15 \mathrm{~min}$ are selected. Even though many of those CPR measurements are outside the MHS pixel, all are stored in the collocated dataset, because the MHS pixel size is a function of the scan angle, and some applications may allow for the CPR pixel to be (just) outside the MHS measurement. Also, it is cheap to select a subset of collocations, but to find pixels slightly further away than the initial criterion, the algorithm would need to be rerun.
For each collocation and for each sensor (CPR, MHS, HIRS and AMSU-A), we store the location (lat/lon), the measurement time, the time of the first measurement in the file (to help finding the file containing the measurement) and the location of the point inside the datafile (row/column). We also store the distance of each centerpoint to the CPR centerpoint, and the time difference (MHS time minus CPR time). With this information, one can find exactly which of the CPR pixels fall inside the MHS pixels, possibly considering the sensor spatial response function.

For the second dataset, we collect the CPR pixels in an MHS pixel and calculate the number of CPR measurements, the average, the standard deviation and the coefficient of variation (standard deviation divided by mean) of the IWP product. Here, we choose a circular MHS pixel area with a radius of $7.5 \mathrm{~km}$, so we are certain that the CPR pixels are contained by the MHS pixel independently of the scan angle. We also note the cloud fraction, defined as the number of CPR pixels with at least $1 \mathrm{~g} \mathrm{~m}^{-2}$ of ice divided by the total number of CPR pixels inside the MHS measurement.

\subsubsection{Comparison with Nagle and Holz (2009)}

The method described above is quite different from the method described by Nagle and Holz (2009), henceforth referred to as "NH".

NH divide the two instruments to be collocated into a master and a slave, where the small slave observations are projected on the large master footprint. They find the location of the satellites as a function of time (forward navigation) and "estimate the time at which a slave satellite passes abeam of a master FOV on the surface" (inverse navigation). They then calculate simultaneous nadir observations (SNO), when two satellites pass over any point on the ground within a certain time window. For this calculation, NH use an orbital prediction model. They search the scan lines around the SNO for overlap with the master FOV. NH assign weights to each of the slave observations based on the sensor spatial response function of the master.

$\mathrm{NH}$ claim that their method works for any combination of satellite, aircraft and ground observations. However, a scanning instrument might very well collocate with a ground observation without any SNO if the measurement is strongly off-nadir. For (near)-parallel orbits, this can be the case between different satellites as well. In fact, at one point $\mathrm{NH}$ "presuppose that the two orbital planes are not nearly coincident".

$\mathrm{NH}$ use the satellite position to calculate the projected sensor spatial response function on the Earth surface. We use an expression from Bennartz (2000) to calculate the size of the pixel, and we do not presently consider the sensor spatial response function. 
$\mathrm{NH}$ was designed to be computationally efficient and may very well be faster than our method. However, our method is conceptually simpler than NH. Our method does not require any forward or inverse navigation. It finds collocations regardless of the presence of simultanuous nadir observations. For some applications, only simultanuous nadir observations are of interest; in this case, $\mathrm{NH}$ and our method should give the same result.

The processing of slightly more than two years of data from CloudSat and five AMSU/MHS sensors with our methods took around two weeks of computer time on a powerful workstation (Intel Xeon Dual Quadcore 3.20 GHz, 16 Gigabyte Random Access Memory (RAM)). Most of this time was due to transferring files over the network and decompressing them. We did not carry out a comparison of speed and results using a common set of source data.

\subsection{Collocation statistics}

We have located collocations for the period between 15 June 2006 13:12 and 4 October 2008 10:34. For the year 2007, we have found 124822977 collocations between the NOAA-18 MHS and the CloudSat CPR, where the maximum distance between MHS and CPR centre points did not exceed $15 \mathrm{~km}$ and the time difference between MHS and CPR measurements was limited to $15 \mathrm{~min}$. With a maximum distance of $7.5 \mathrm{~km}$ and counting the MHS pixels, the number of collocations reduces to 2669135 . If only tropical nadir points are selected (within 30 degrees of the equator, within 1 degree of nadir), around $1 \%$ or 26410 MHS pixels remain.

Figure 3 shows the latitudes at which collocations occur between the CloudSat CPR and the MHS/AMSU-B on different satellites. It shows that only the NOAA-18 MHS has collocations with the CPR globally. This is due to the fact that the LTAN of the NOAA-18 (13:39) is always similar to the CloudSat LTAN (13:30-13:45). NOAA-18 is near the ATrain constellation and thus near CloudSat, because CloudSat is part of the A-Train. All other POES considered in this study have collocations with CloudSat CPR only near the poles.

Figure 4 shows at which angles and latitudes the collocations occur. At the equator, no nadir collocations with a time difference of less than one minute occur. Rather, the viewing angle is sligthly off-nadir. If two satellites pass through the same place in space $^{2}$ with one minute in between, the Earth rotates so their subsatellite points are roughly $1 \mathrm{~m} / 24 \mathrm{~h} \cdot 40075 \mathrm{~km} \approx 27.8 \mathrm{~km}$ apart. For a NOAA18 altitude of $850 \mathrm{~km}$, the viewing angle then needs to be $\tan ^{-1}(27.8 / 850)=1.9^{\circ}$. In reality, the satellites do not pass through the exact same point, and the viewing angles for collocations within one minute are slightly larger. The CloudSat has a slightly lower inclination than NOAA-18, so for a

\footnotetext{
${ }^{2}$ The same place in space in an Earth-centered inertial reference system.
}

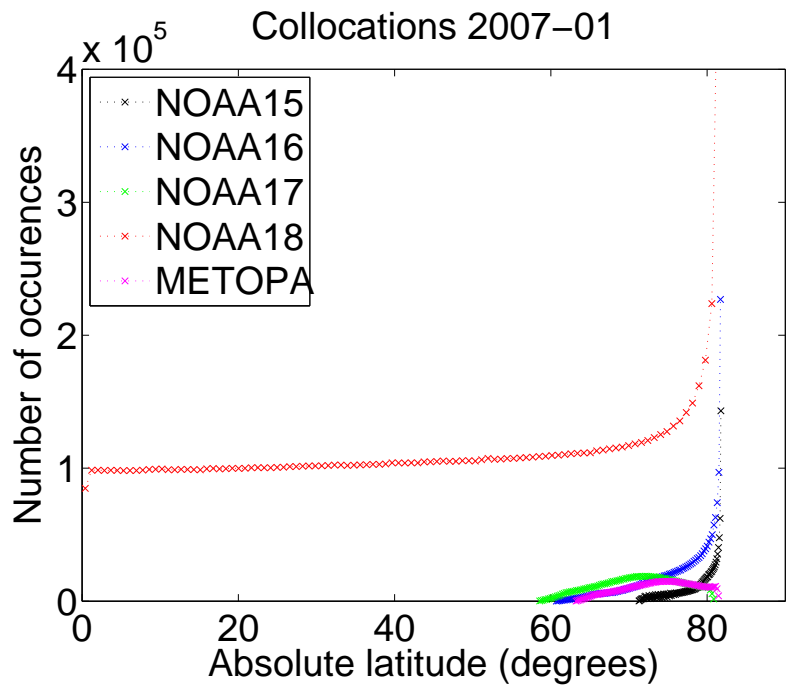

Fig. 3. A histogram of the number of collocations between the CloudSat CPR and the AMSU-B or MHS sensors on various satellites in January 2007. The maximum distance for a collocation is $15 \mathrm{~km}$; the maximum time between the collocated measurements is $15 \mathrm{~min}(900 \mathrm{~s})$. The number of collocations refers to the number of CPR pixels collocating with an AMSU-B or MHS pixel.

collocation to occur, NOAA-18 has to look to the left when it reaches its northernmost point and to the right when it reaches its southernmost point.

CloudSat and NOAA-18 are in some sort of "orbital resonance", as shown in Fig. 5, showing the collocations in January 2007. Figure 5 shows a time series of the number of collocations per hour, where the upper left is 1 January, 00:00-00:59 and the lower right is 31 January, 23:00-23:59 (inclusive). The figure shows a collocation pattern with a 56-h period: $16 \mathrm{~h}$ with collocations, $40 \mathrm{~h}$ without.

\subsection{Sampling effects}

As shown in Fig. 1, an MHS footprint is an order of magnitude larger than a CPR footprint. The smallest MHS pixel is the nadir-viewing pixel, which has a diameter of $16 \mathrm{~km}$. The CPR pixel can be approximated by an ellipse of 1.3 by $1.7 \mathrm{~km}^{2}$. It covers at most $0.65 \%$ of the area an MHS pixel:

$\frac{A_{\mathrm{CPR}}}{A_{\mathrm{MHS}}}=\frac{\pi \frac{1.3}{2} \frac{1.7}{2}}{\pi\left(\frac{16}{2}\right)^{2}}=0.0065=0.65 \%$

Many CPR measurements fit in one MHS measurement. Since the CPR is not a scanning instrument, CPR pixels never fill an MHS pixel completely. In the best case, a nadir MHS pixel contains around 15 CPR pixels (or only 13 when we limit the collocations to CPR pixels within $7.5 \mathrm{~km}$ of the MHS centrepoint). The total area is less than $15 \cdot 0.65 \%=9.75 \%$ because of the overlap between subsequent CPR pixels. Usually, the CloudSat ground track does 


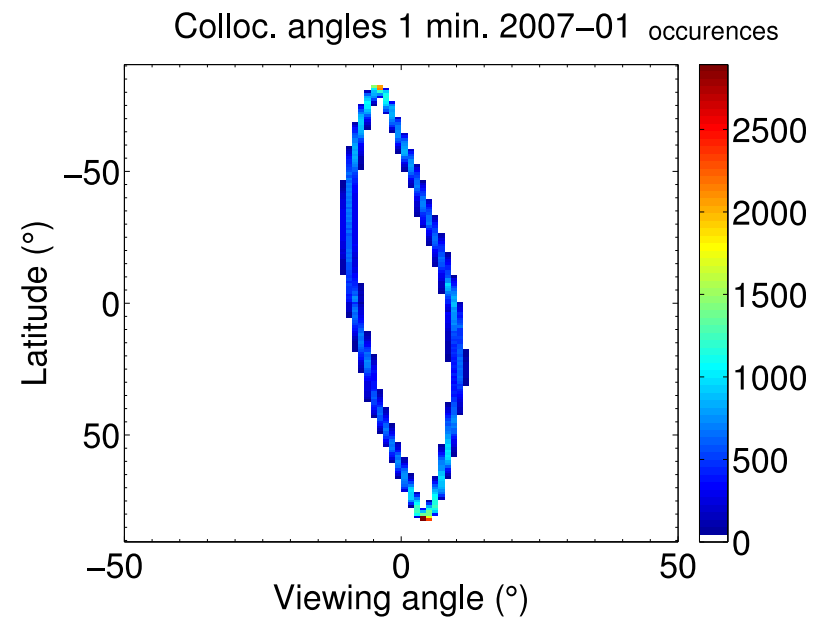

Fig. 4. This two-dimensional histogram shows at which angles the collocations between the NOAA-18 MHS and the CloudSat CPR occured in January 2007. The figure shows collocations with a maximum time interval of $1 \mathrm{~min}$.

not pass through the centre of the MHS pixel, and the situation is worse. Hence, sampling effects need to be taken into consideration.

A collocation is considered representative, or good, if the CPR IWP statistics for the area covered by CPR are the same as the statistics of a hypothetical CPR IWP covering the full MHS pixel.

CPR pixels inside the MHS pixel have the same statistics as they would if they would fill the entire MHS pixel. Whether the collocation is representative cannot be known exactly, because high-resolution information on the part of the MHS pixel not covered by CPR pixels is not available in this approach. However, we can look at some indicators to make an educated guess as to how well the CPR pixels represent the MHS pixel.

Figure 6 shows three graphs that give some insight in the sampling error. The MHS pixel is assumed to be circular with a radius of $7.5 \mathrm{~km}$.

In Fig. 6a we can see that most collocations contain a relatively large number of CPR pixels, but many do not. When the number of CPR pixels inside the collocation is small, the CPR pixels are close to the MHS footprint edge and poorly represent the MHS pixel. The highest number of CPR pixels inside a MHS pixel occurs when the CPR groundtrack passes close to the centre of the MHS footprint. This is the optimal case.

Figure $6 \mathrm{~b}$ shows a histogram of the coefficient of variation of the CPR IWP product for the CPR pixels within $7.5 \mathrm{~km}$ of the MHS centrepoint. A small coefficient of variation corresponds to a homogeneous cloud. The more homogeneous the cloud, the more representative the CPR pixels are for the complete MHS footprint area. We use the coefficient of variation rather than the standard deviation because the standard

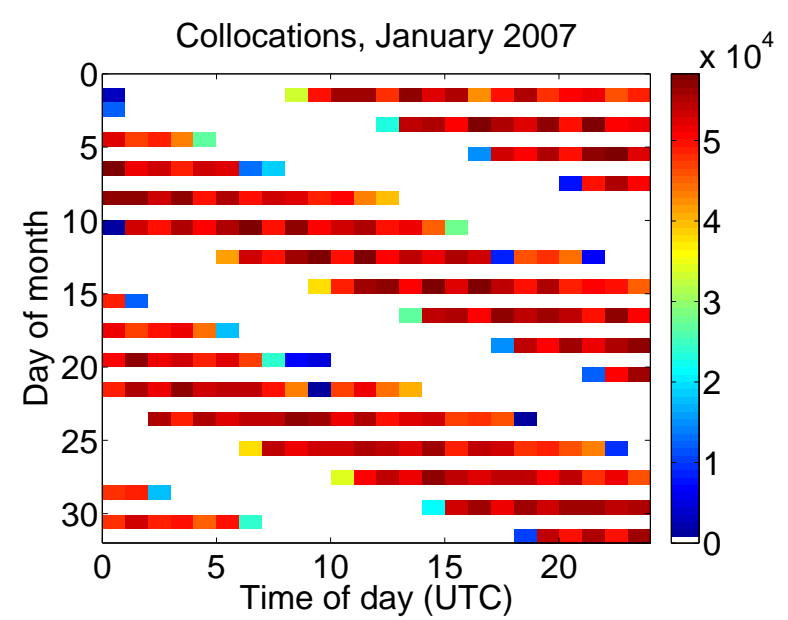

Fig. 5. Number of collocations per hour in January 2007. The vertical axis shows the day of the month. The horizontal axis shows the universal time.

deviation is likely to be much larger for clouds with a high IWP than for clouds with a low IWP. Selecting collocations based on the standard deviation would throw away many of the measurements with high IWP. The coefficient of variation is largest when some CPR pixels measure a strong cloud and others do not measure any cloud at all. This indicates the presence of a strong, localised cloud, which significantly reduces our trust in the representativeness of the CPR pixels.

In Fig. 6c, the distribution of CPR inside MHS is shown for three cases. The red dots show a case with an extremely high coefficient of variation (2.106; note in panel (b) that a coefficient of variation larger than 2 is so rare that it is not visible in the histogram). Since a thick cloud that is only $1 \mathrm{~km}$ in diameter is unlikely, this happens usually when the cloud is just on the edge of the MHS pixel. In either case, the CPR pixels do probably not share the same statistics as the MHS footprint and the collocation is not useful. The green dots show a case with a very low coefficient of variation $(0.017$; cases where all CPR pixels have the same nonzero measurement and the coefficient of variation is 0 occur as well, but the IWP value tends to be $1 \mathrm{~g} \mathrm{~m}^{-2}$ so it would not be visible in this graph). The portion of the cloud imaged by CPR has a roughly constant IWP of around $70 \mathrm{~g} \mathrm{~m}^{-2}$. It is quite likely that the rest of the MHS pixel looks similar. The example in blue shows a collocation with a coefficient of variation of 0.354 .

When the criteria discussed above are applied, sampling effects are reduced and a large number of collocations remain. 

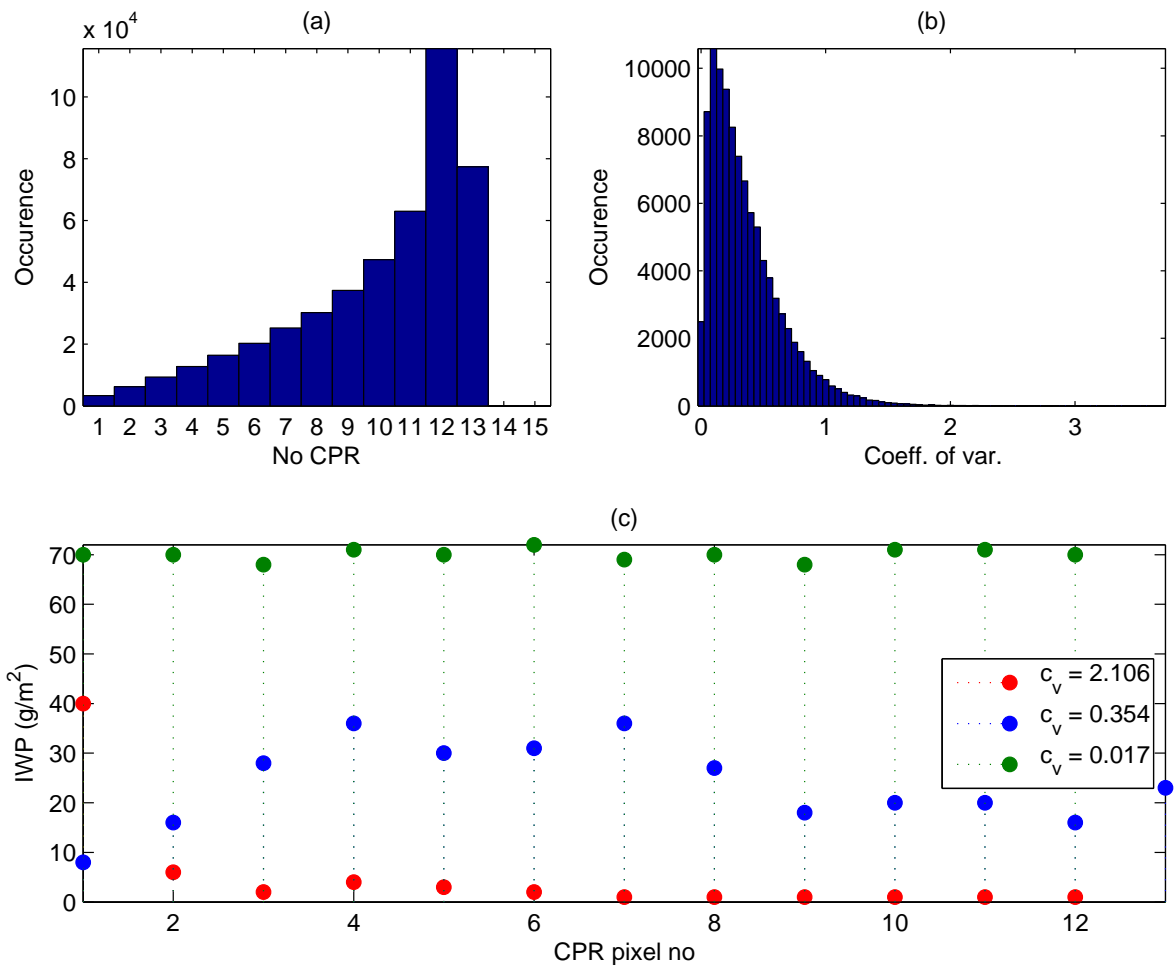

Fig. 6. Some collocation properties for 2007. (a) shows a histogram of the number of CPR pixels that fit inside a MHS pixel (circular with a $7.5 \mathrm{~km}$ radius). (b) shows a histogram for the coefficient of variation of all collocations that contain only cloudy pixels. (c) shows examples of how CPR IWP may be distributed inside a MHS pixel. See text for a discussion.

\section{Applications}

Collocations can be used in many different ways. This section presents some possible applications of collocations between CloudSat CPR and NOAA-18 MHS. Three examples are explored in the following subsections. This section is meant to show what can be done with such a collocated data set and does not provide a comprehensive study of the different applications.

\subsection{Comparison with NESDIS IWP}

Various algorithms exist to determine IWP from microwave radiometer measurements (Liu and Curry, 2000; Zhao and Weng, 2002; Weng et al., 2003). The National Environment Satellite, Data and Information Service (NESDIS) publishes an operational IWP product from MHS measurements in the Microwave Surface and Precipitation Products System (MSPPS). Zhao and Weng (2002) assume spherical ice particles and calculate the effective particle diameter from the ratio between the scattering at $89 \mathrm{GHz}$ and $150 \mathrm{GHz}$. They assume a constant bulk volume density and calculate the IWP from this. They also discuss how errors propagate in the retrieval algorithm, but no discussion of systematic error and no validation for the NESDIS MSPPS IWP was found in this paper, nor elsewhere in the literature. Waliser et al. (2009) find a dry bias in the NESDIS IWP product. They explain this from the Zhao and Weng (2002) screening criteria and the MHS insensitivity for ice particles smaller than $0.4 \mathrm{~mm}$.

CloudSat IWP has a systematic uncertainty of up to $40 \%$ (Austin et al., 2009). Judging from the available data, the detection limit for CloudSat IWP is $1 \mathrm{~g} \mathrm{~m}^{-2}$.

Figure 7 shows a comparison of the NESDIS MSPPS IWP with the CloudSat IWP. It shows that the NESDIS IWP is systematically smaller than the CPR IWP. For many nonzero CloudSat measurements, the NESDIS IWP is zero. This is because thin clouds are (almost) transparent for microwave radiation in the frequencies at which MHS operates (Greenwald and Christopher, 2002). For some NESDIS IWP measurements, the CloudSat IWP is zero. This happens due to the different footprint sizes. The MHS footprint is much larger than the CPR footprint. A cloud that does not cover a complete MHS pixel may be missed by the CPR (see Sect. 3.3).

MSPPS IWP is systematically lower than CPR IWP by approximately 70-90\%. Austin et al. (2009) estimate the CPR accuracy to $40 \%$, based on a retrieval blind comparison study by Heymsfield et al. (2008), which was based on simulated radar observations for ice particle data from aircraft in-situ measurements. While the profiles considered in that study may not be representative for all atmospheric cases, we can 
still consider the CPR data to be considerably better validated than the MSPPS data. It is therefore likely that the difference reflects a real low bias in the MSPPS data. This is partly a fundamental problem, because of the transparency of thin clouds to radiation at MHS frequencies. However, MSPPS underestimates the IWP for thick clouds as well. A more accurate IWP product based on microwave measurements is probably possible. One way to obtain such a product is by using a neural network, described later in the article.

\subsection{Comparison of BT-IWP relations}

As a second application example, we investigate the relation between the MHS channel 5 brightness temperature and the associated Ice Water Path for two different datasets. The first dataset consists of the collocations, providing a mapping between brightness temperatures and independent IWP. The second dataset consists of a mapping generated from 30000 synthetic atmospheres as described below. Note that this mapping predates the collocated measurements. Rydberg et al. (2009) use this method to derive IWC from the Sub-Millimetre Radiometer (SMR) on the Odin satellite. It can potentially be used to derive IWP from MHS.

Atmospheric states, including clouds, are generated following the procedure described by Rydberg et al. (2009), and a brief overview is given here. Cloud states are generated in a series of steps, where two-dimensional (2-D) radar reflectivity fields from the Cloud Profiling Radar on-board CloudSat serve as the basis for obtaining realistic cloud structures. Orbit sections of CloudSat data (with a resolution of $\sim 250 \mathrm{~m}$ in vertical by $2 \mathrm{~km}$ along the scan line) are transformed to 3-D by inputting those into a stochastic iterative amplitude adjusted Fourier transform algorithm (Venema et al., 2006). This algorithm generates surrogate 3-D radar measurement fields with the same spatial resolution as the original fields.

Cloud microphysical fields are generated in such a way that the surrogate 3-D radar reflectivity fields are conserved. This is done by assuming that spherical ice particles can be used to represent the single scattering properties of natural occuring ice particle populations. We lack information about the true shape of the ice particles, which is different for different cloud types, and the most generic assumption is to assume spheres. This is also the assumption made by Austin et al. (2009) for the CloudSat CPR IWP retrieval. The accuracy of this approximation is difficult to assess, because the true microphysical parameters are unknown. Furthermore, the cloud ice particle size distribution (PSD) parameterisation derived by McFarquhar and Heymsfield (1997) (hereafter MH97) is assumed to be the best representation of the tropical mean PSD. MH97 depends on temperature and ice water content (IWC), and is used to map radar reflectivity fields to IWC and PSD fields. However, it should be clear that local PSD may deviate significantly from MH97. For temperatures above $273 \mathrm{~K}$, clouds are assumed to consist

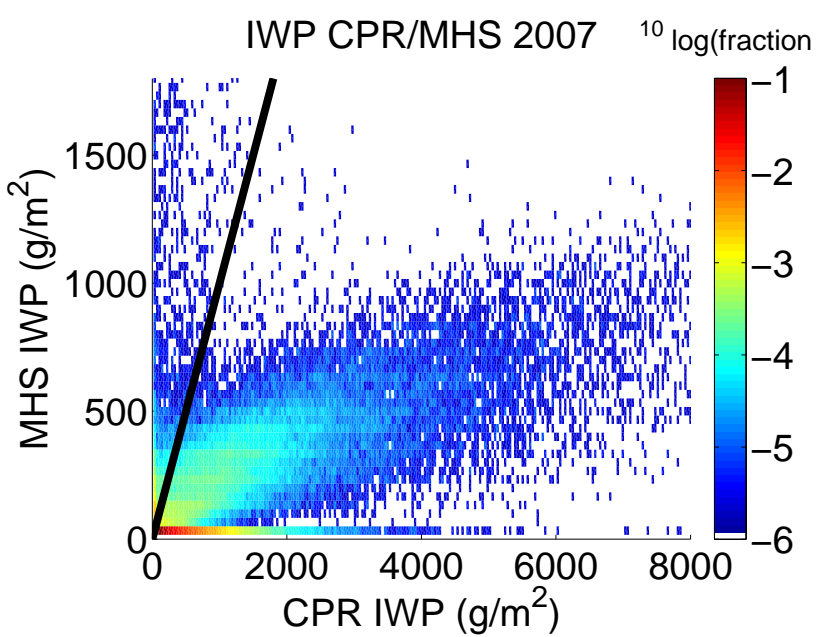

Fig. 7. Two-dimensional histogram of CloudSat CPR Ice Water Path (averaged over an AMSU pixel) and NOAA NESDIS MSPPS IWP, for all collocations in the year 2007. The figure is similar to a scatter plot, but it shows the density of points rather than the actual points. Only measurements where either value is nonzero are shown. The black line shows the ideal case. The colour scale is logarithmic. See text for a discussion.

entirely of spherical water particles and the PSD of stratus cloud derived by Deirmendjian (1963) is used.

Weather data (temperature, humidity, and pressure) and ozone information, originating from the European Centre for Medium-Range Weather Forecasts (ECMWF), are obtained from the CloudSat auxiliary data archive (ECMWF-AUX). ECMWF-AUX contains ECMWF state variable data interpolated to each CPR bin. These fields are handled as described by Rydberg et al. (2009) in order to have a realistic variability that accounts for variations on scales not resolved by ECMWF.

Radiative transfer simulations of nadir viewing AMSU-B channel 20 (corresponding to MHS channel 5) are performed using version 1.1 of the Atmospheric Radiative Transfer Simulator (ARTS). This is a development of the first version, ARTS-1 (Buehler et al., 2005), where two scattering modules, a discrete ordinate iterative method (Emde et al., 2004) and a reverse Monte Carlo algorithm (Davis et al., 2005) have been implemented to solve the polarised radiative transfer equation. The Monte Carlo module is used and the 3-D variability of the atmosphere is fully considered in the radiance simulations. The lower and upper sidebands of AMSU-B channel 20 are represented by single frequencies of 176.01 and $189.91 \mathrm{GHz}$, respectively. For a diverse set of atmospheric profiles, the root mean square error between this approximation and a setup with a finer frequency grid is $0.020 \mathrm{~K}$. The instrument antenna spatial response function is assumed to be a 2-D Gaussian with a full-width half-power beamwidth of $1^{\circ}$ in both dimensions. Pencil beam simulations with a grid spacing matching the 
atmospheric states horizontal resolution are performed. After the antenna weighting the precision of the simulations is better than $0.5 \mathrm{~K}$. The IWP is extracted along each pencil beam where radiative transfer simulations are performed. The atmospheric scenario has a higher spatial resolution than AMSU-B, so the simulated IWP are weighted according to the antenna pattern to obtain the AMSU-B IWP.

Figure 8 shows a comparison between the two relations. We average the CPR IWP over the MHS pixel, and select a subset of collocations. For the collocations, only measurements that are within 20 degrees of the equator are used, in order to prevent a signal from the surface (Buehler and John, 2005). Only collocations where the MHS measurement is within 5 degrees of nadir are used, so that no significant limb effect occurs. Finally, collocations are selected where all CPR pixels are cloudy and the coefficient of variation is smaller than one, for reasons discussed in Sect. 3.3 above.

The figure shows AMSU-B channel 20 or MHS channel 5 brightness temperature as a function of the IWP (logarithmic) for the two different datasets. In blue are the collocated measurements (MHS channel 5 and CPR IWP). The red boxes show simulated radiances for generated atmospheric states (AMSU-B channel 20 and generated IWP).

The figure shows that both datasets have largely the same statistical features. For IWP up to around $100 \mathrm{~g} \mathrm{~m}^{-2}$, the effect on the brightness temperature is minimal, because thin clouds are not resolved at MHS channels 3-5 frequencies (Greenwald and Christopher, 2002). For higher values of IWP, the brightness temperature decreases logarithmically as a function of IWP. For IWP $>100 \mathrm{~g} \mathrm{~m}^{-2}$, the simulated brightness temperatures are slightly higher than the observed ones.

The microphysical assumptions for the generated atmospheric states are based on MH97, which differ from the assumptions in the CloudSat retrieval. This might contribute to the observed differences.

Overall, the variability in the simulated brightness temperatures is smaller than the variability in the observed brightness temperatures. This effect is stronger for higher values of the IWP. Several factors may contribute to this discrepancy. The CPR pixels are much smaller than the MHS pixels, so the measured value is averaged over a smaller area. If a small, concentrated cloud exists inside a MHS pixel, the CPR might either see it, in which case it measures a higher IWP than the MHS, or it might miss it, so it measures a lower IWP. This adds to the variability. Additionally, the generated atmospheric states might not fully resolve the natural variabily of cloud microphysical parameters and of atmospheric temperature and humidity.

\subsection{Developing a retrieval using neural nets}

An artificial neural network (ANN) is an interconnected assembly of processing units called neurons (e.g. Jiménez et al., 2003). Neural nets are widely used to statistically char- acterise the mapping between radiometric measurements and related geophysical variables (e.g. Krasnopolsky, 2007). We use an ANN to characterise the mapping between MHS radiances and the CPR IWP, and then use the trained ANN to retrieve IWP from the MHS measurements. We call this retrieval MHS-CPR IWP.

MHS-CPR IWP has both advantages and disadvantages compared to other retrieval approaches. One can use a neural network with simulated rather than measured radiances, or one can use a more classical retrieval method. As we use the collocated measurements, an advantage is the relative simplicity; there is no need for a potentially complicated radiative transfer model with many possible sources of error. On the other hand, the collocations approach may introduce a number of errors, as discussed in Sect. 4.3.1. However, an MHS-CPR IWP can complement the other existing retrieval methods. The retrieval quality can never become as good as CloudSat, but the spatial and temporal coverage will be much larger.

The neural network approach described below is in the exploration phase and will be developed further.

We select a subset of collocations that provide a relatively homogeneous dataset. The subset is restricted to pixels over ocean within 20 degrees of the equator, because a warm (and humid) atmosphere prevents the MHS from getting a signal from the surface (Buehler and John, 2005). Due to these restrictions, the neural network is only applicable to the tropics. A strongly off-nadir measurement is colder due to the limb effect (Buehler et al., 2004). For the training, we restrict ourselves to measurements within 5 degrees of nadir. This avoids the need to compensate for this effect (described below). The neural network works for nadir measurements or measurements where the limb effect is compensated.

As discussed in Sect. 3.3, the MHS measurement compromises a larger area than the CloudSat measurement, even when we average the CPR pixels inside an MHS pixel. If a small, strong event is present inside an MHS pixel, the CloudSat might miss it completely or measure exactly this event. In both cases, the observed MHS radiance is the same, but the CPR IWP can vary considerably. For that reason, we select only homogeneous measurements: the collocation shall contain at least ten CPR pixels, all measuring at least $1 \mathrm{~g} \mathrm{~m}^{-2}$, and the standard deviation shall not exceed the mean value. The selection of only "cloudy pixels" for the training leads to a wet bias, because the neural network tends to the mean state if it has insufficient information from the input. We want to explore the effect of adding HIRS channels on the neural network retrieval. Hence, we choose collocations where at least five CPR pixels are within $10 \mathrm{~km}$ of the nearest HIRS pixel.

Finally, only collocations where the time interval is at most ten minutes are selected.

For the year 2007, we find 2627 collocations that meet the criteria described above. 


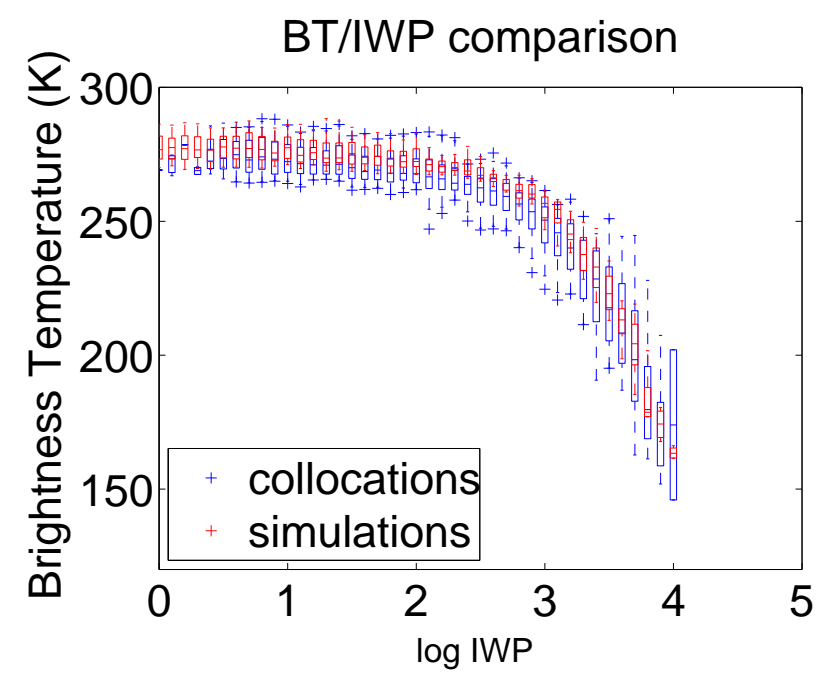

Fig. 8. Modified boxplot of Ice Water Path and MHS channel 5 or AMSU-B channel 20 brightness temperature. Radiances are binned in ${ }^{10} \log$ IWP bins with a width of $0.1 \mathrm{log} \mathrm{g} / \mathrm{m}^{2}$. In each bin, the median brightness temperature is shown as a horizontal line. The upper and lower bars of the rectangle show the 1st and 3rd quartile (25th and 75th percentile). From the rectangles, dashed lines connect to the 1st and 99th percentile. All other points are defined as outliers and plotted as pluses. Collocations are shown in blue and simulations are shown in red.

For the neural network calculations, we use the MATLAB Neural Network toolbox V6.0.1 (R2008b). The collocations are divided in $60 \%$ training, $15 \%$ testing and $25 \%$ validation. MHS channels 3, 4 and 5 are the inputs. As a target, we choose the log IWP which was found to work better than the ordinary IWP. The transformation is reversed after the application of the neural network. Throughout the process, CPR IWP is assumed to be the truth. The training is considered to be finished if the error with the testing data increases for fifteen consecutive iterations. After training, we store a neural network that we can then use for our retrieval.

To compensate for the limb effect, we correct the brightness temperatures before we input them to the network. For each viewing angle and channel, the mean brightness temperature is calculated. We use only tropical measurements (within 30 degrees of the equator) to prevent an angledependent signal from Antarctica, which is mainly seen by one side of the scan. The limb effect is minimal for the two viewing angles closest to nadir. The average brightness temperature for those angles is the reference. The limb effect can be quantified by the difference between the reference brightness temperature and the mean brightness temperature for a certain viewing angle. We compensate for the limb effect by adding this difference to all measurements for this viewing angle.
In Fig. 9 we show an example of how a NN IWP product might look like. The data is for 1 January 2008. The left panels show the MHS brightness temperatures between 08:56 and 19:02 UTC, the right panel shows the IWP retrieved by the neural network.

\subsubsection{Error analysis}

Four sources of error can be identified: (a) The CPR IWP uncertainty is up to $40 \%$ (Austin et al., 2009). This propagates directly into the MHS-CPR IWP. (b) Collocation mismatches add noise to the training data, as discussed in Sect. 3.3. This may or may not result in an error in the MHS-CPR IWP (noise in the input data need not change the best fit). (c) The inversion from MHS data inherently has a limited accuracy, leading to a significant uncertainty in the MHS-CPR IWP. (d) The MHS has a radiometric noise of up to $0.55 \mathrm{~K}$ and might suffer from calibration errors.

Figure 10 shows a scatter plot between CPR IWP and collocated MHS-CPR IWP. Both axes are logarithmic. (a) and (d) do not contribute to the variability seen here. MHS-CPR IWP could still perfectly reproduce MHS-CPR IWP even considering the MHS radiometric noise, because this noise is part of the training data. If it would do so, CPR IWP might still be off by $40 \%$ compared with the true atmospheric IWP, but Fig. 10 would not show variability.

The variability is consistent with simulations similar to the ones described in (Jiménez et al., 2007). Since those simulations did not use collocations, the dominant source of the variability in Fig. 10 is likely to be the inversion error (c).

For low IWP, the network exhibits a wet bias. Thin clouds are (almost) completely transparent at MHS frequencies (Buehler et al., 2007), so with only those measurements, there is no information for thin clouds. With no information, the neural network tends towards the mean state. Since only cloudy CPR pixels were used for the training, this explains the wet bias.

Figure 11 shows the neural network sensitivity to MHS radiometric noise. A subset of tropical nadir measurements for 2007 are selected. For practical reasons, this subset consists of the MHS measurements for where collocations could be found; however, as the CloudSat values are not used for this figure, those measurements are effectively a sample of all MHS measurements for 2007. The figure shows the mean fractional IWP error as a function of IWP and input noise. For this figure, the neural network is applied twice. First, the unperturbed input data (MHS brightness temperatures for channels 3, 4 and 5) are fed into the ANN. This gives an unperturbed IWP for each measurement. Then, we add gaussian noise, starting with $\sigma=0.1 \mathrm{~K}$, to the input data, and feed this perturbed data to the ANN. This results in a perturbed IWP denoted by IWP. For each collocation, the fractional error is calculated as $\left|\frac{\text { IWP }}{\mathrm{IWP}}-1\right|$. Those fractional errors are divided into bins according to the unperturbed IWP value. 


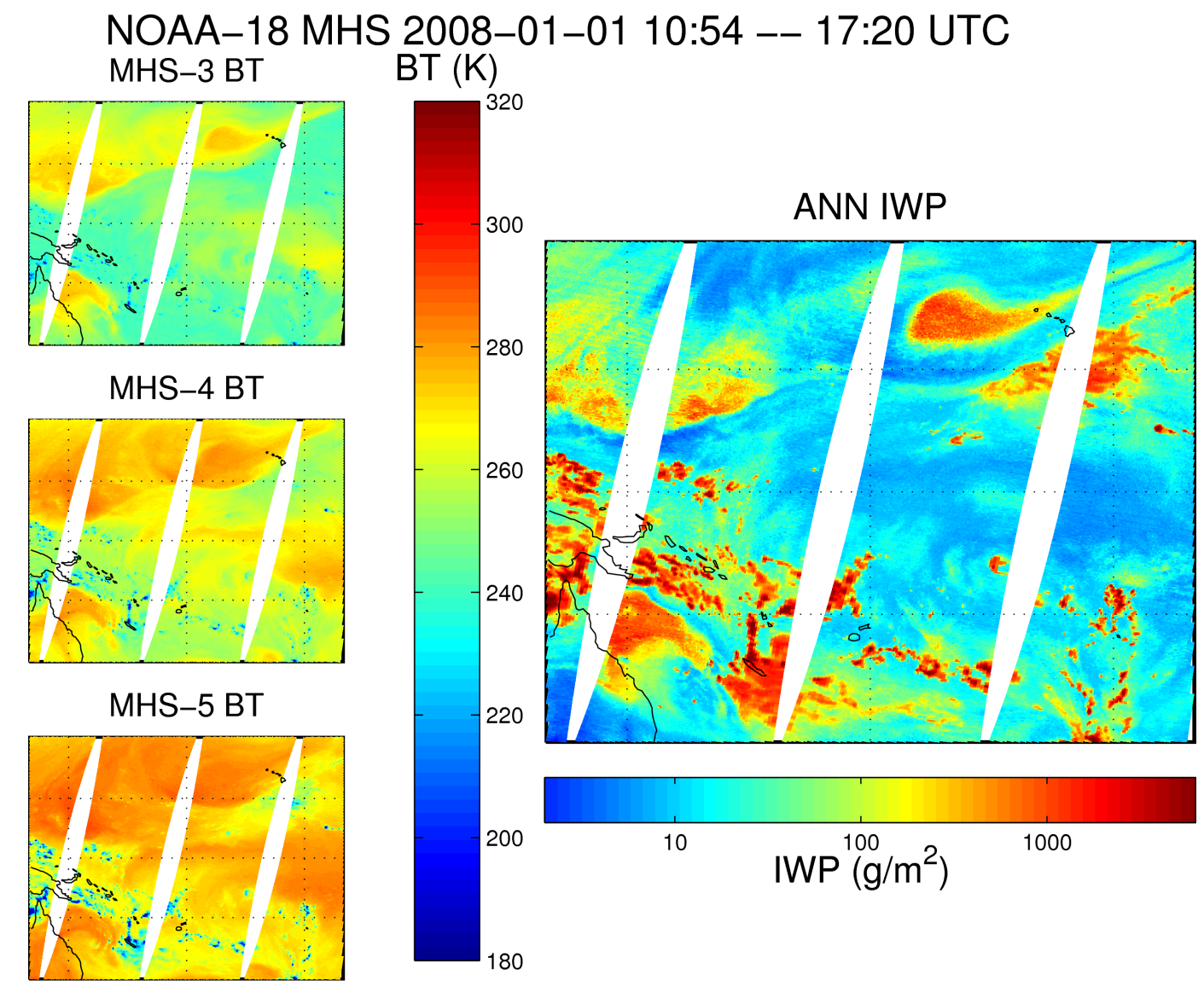

Fig. 9. The neural network (see text) can be used to retrieve IWP from radiances. The figure shows observations by NOAA-18 in the descending node on 1 January 2008 between 10:54 and 17:20 UTC (local time during the night). The left panels show the brightness temperatures as observed by the MHS channels 3-5. The right panel shows the IWP as generated with the neural network as described in the text. Cold areas in the left panel correspond to wet areas in the right panel.

For each bin, we calculate the mean fractional error. This process is repeated for higher values of $\sigma$, up to $\sigma=2.0 \mathrm{~K}$, taking steps of $\sigma=0.1 \mathrm{~K}$.

Unsurprisingly, Fig. 11 shows that a higher input noise results in a higher error in the output. This effect is linear. The mean fractional error as function of IWP is less straightforward. The error is largest for IWP values of around $100 \mathrm{~g} \mathrm{~m}^{-2}$ and smaller for values that are either larger or smaller. This can be explained as follows. For small IWP, a small perturbation in the brightness temperatures has little influence on the IWP. The ANN does not interpret the brightness temperature noise as IWP. This is in line with the observation that thin clouds are transparent to the frequencies at which MHS operates (Greenwald and Christopher, 2002), and can also be seen in Fig. 8. For large IWP, MHS channels 3-5 will observe large depressions in brightness temperature, and a $2 \mathrm{~K}$ noise is much smaller than the signal, so its effect on the output is also small. However, for intermediate values of IWP, around $100 \mathrm{~g} \mathrm{~m}^{-2}$, the noise is of a similar order of magnitude as the signal, and the ANN is quite sensitive to input noise. The actual radiometric noise for MHS depends on the channel, but is always below $0.55 \mathrm{~K}$ (Kleespies and Watts, 2007). This means that radiometric noise is unlikely to be a dominant error source for this kind of IWP retrieval method.

\subsubsection{Adding HIRS}

Thin clouds are not visible by MHS channels 3-5 because the effect of ice clouds on microwave radiation at those frequencies is relatively small. In the infrared, the situation is different: even a small cloud has an observable effect, but an infrared sensor does not see the difference between a medium cloud and a thick cloud, because the sensor is saturated quickly (Jiménez et al., 2007). Hence, we can expect the retrieval quality to improve if we combine infrared and microwave measurements.

Figure 12 shows a scatter plot similar to Fig. 10, but with additional HIRS channels 8 and 11 (chosen for their clear cloud signal). The number of collocations used for the neural net remains the same, because we already preselected the 


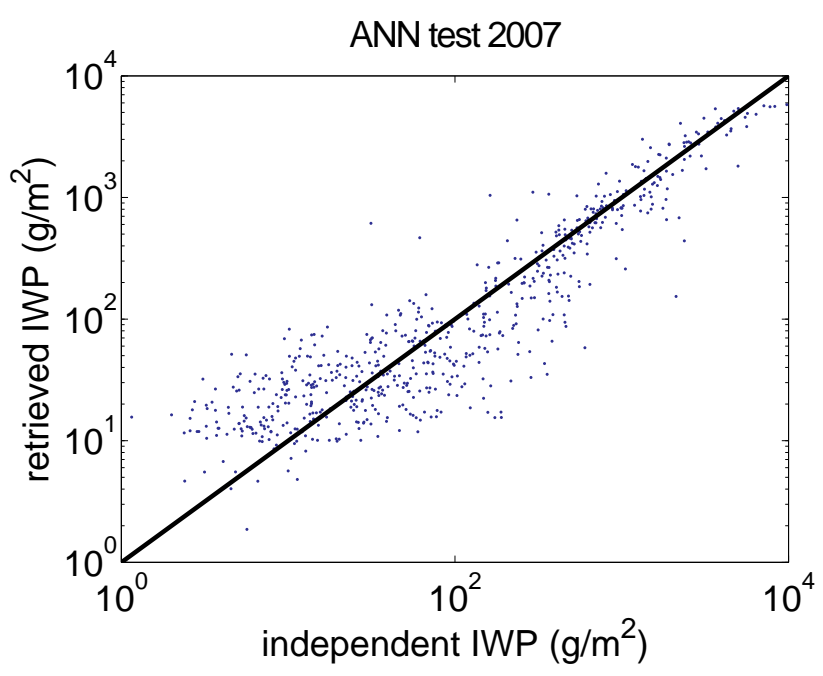

Fig. 10. Scatter plot showing the performance of the ANN using MHS channels 3 to 5. The retrieved IWP is plotted against the input IWP.

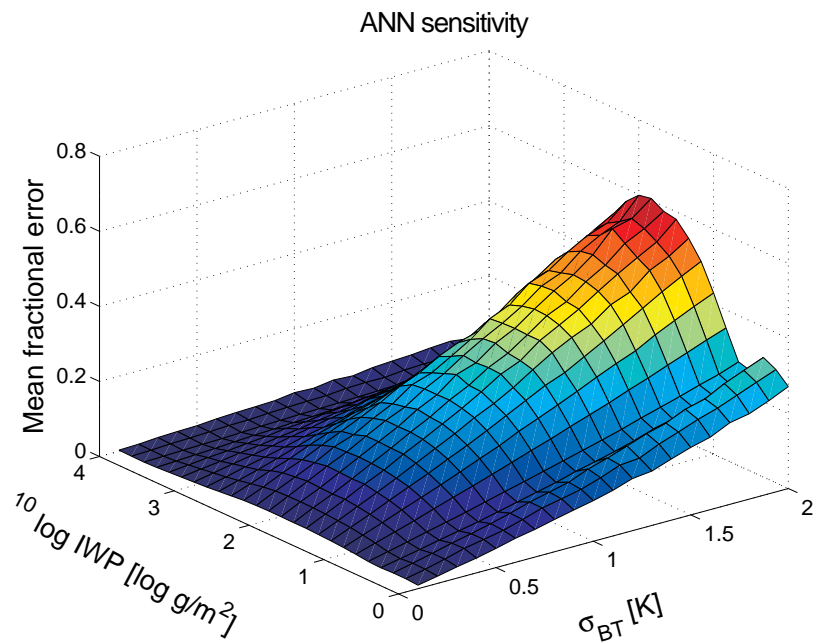

Fig. 11. ANN sensitivity to errors in the input brightness temperatures. Here, only the MHS channels are used as input to the ANN. See the text for an explanation and a discussion.

collocations so that at least five CPR pixels are less than $10 \mathrm{~km}$ from the nearest HIRS pixel centerpoint.

By eye, it is hard to see whether there is any improvement gained by adding them.

Figure 13 shows the fractional median error as a function of IWP for both cases. Here, the fractional median error is defined relative to CloudSat, so CloudSat is assumed to be true. The dashed line shows the error for the ANN where the input consists only of MHS channels, the dotted line shows the error for the ANN with an input consisting of MHS channels 3-5 and HIRS channels 8 and 11. For small values of IWP there is an improvement when adding the HIRS chan-

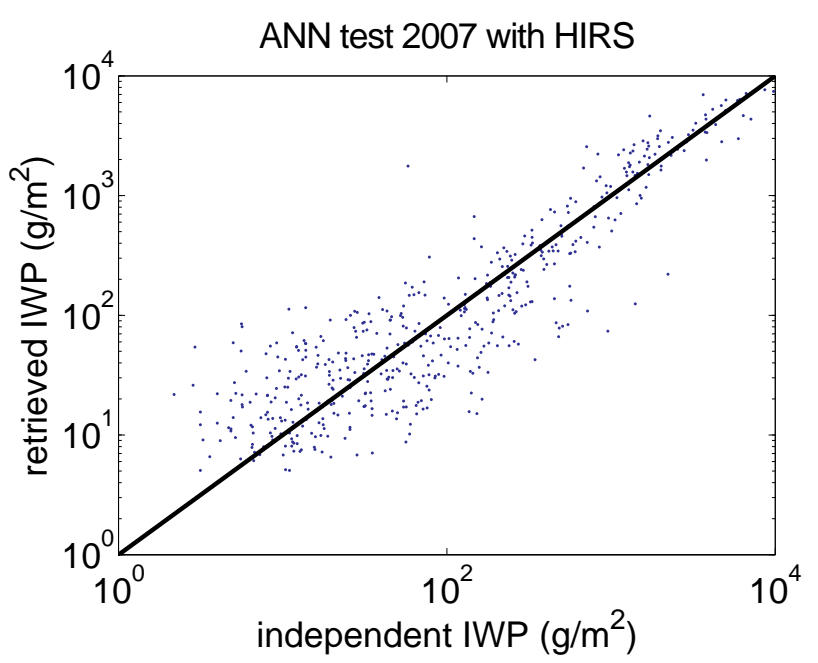

Fig. 12. Scatter plot to show the performance of the neural network, MHS 3-5, HIRS 8 and 11.

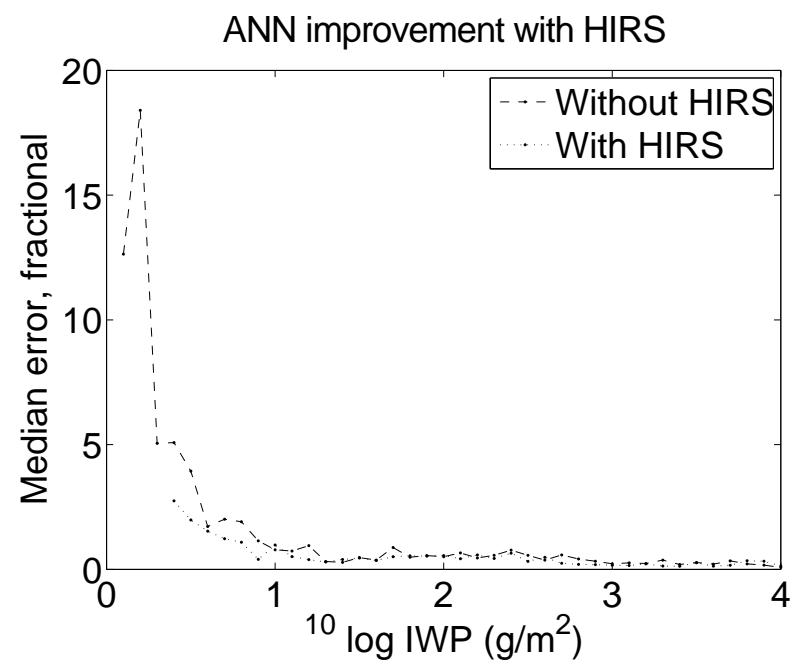

Fig. 13. Comparison of the median fractional error between independent and retrieved IWP, when only MHS channels are used or when both MHS and HIRS channels are used as input to the ANN. The median fractional error is defined as the median of all errors with a certain IWP, where the error is defined as $\left|\frac{\mathrm{IWP}_{\mathrm{NN}^{-I W P}} \mathrm{IPR}_{\mathrm{CPP}}}{\mathrm{IWPR}_{\mathrm{CPR}}}\right|$.

nels. However, the error is still large, since a median relative error of 2 means that the retrieved IWP is on average a factor 2 off. For larger values of IWP, the errors are roughly the same, as expected.

Why the retrieval does not strongly improve when adding HIRS is not yet fully understood. One factor may be the difference in footprint location for HIRS and MHS, even if only collocations with at least 5 CPR pixels in the HIRS pixel are considered. Additionally, HIRS might suffer from the 
beam-filling problem: the sensor may be saturated if only a part of the pixel is cloud-covered, and be unable to tell the difference between a partly cloudy and a fully cloudy pixel. A further investigation is necessary and will be carried out.

\section{Conclusions}

The collocation-finding method described in this work finds many collocations between the NOAA-18 MHS and the CloudSat CPR. Those collocations are frequent and globally distributed. Other POES collocations with CloudSat are limited to the polar areas. Sampling effects due to different footprint sizes need to be taken into consideration.

There are numerous possible improvements to our procedure. The procedure to find the collocations can be refined by considering how the MHS footprint size depends on the scan angle. Even better, one can project the MHS sensor spatial response function onto the surface and calculate a weighted average of the collocated CPR pixels, similar to the procedure described by Nagle and Holz (2009).

In comparison with Nagle and Holz (2009), our algorithm is relatively simple. For example, it does not need satellite position data. It finds collocations even in the absence of simultaneous nadir observations.

Our method was designed for the case where one instrument is scanning and the other has a fixed viewing angle. It also works if both instruments are scanning, but in this case, it is slow and a different method is more suitable. If either satellite is geostationary or both instruments are on the same satellite, more optimised methods may be appropiate. The method does not depend on the nature of the sensor (active, passive) or the footprint size.

The collocations have various applications. They can be used to compare different IWP products. As an example, we have compared the NOAA NESDIS MSPPS MHS IWP product against the CloudSat CPR IWP product. IWP values from the CloudSat CPR were found to be significantly larger than those from the MSPPS. This may be partly attributed because thin clouds are transparent to radiation at MHS frequencies, but since the MSPPS underestimates IWP even for high values, there should be room for improvement.

As a second example, we have compared the IWP-BT relation for our collocations with the one for simulated radiances from synthetic atmospheric cases. The variability in the measured relation was found to be larger than the variability for the simulated relation.

The validation for simulated radiances was performed statistically. A stronger validation would be to simulate the radiances for the exact cases where a collocation exists.

As a final example, we have used the collocations to train an Artificial Neural Network to develop a new IWP product. We have shown that this method is promising. Finally, we have investigated the effect of adding HIRS channels 8 and
11 to such an ANN. Unexpectedly, this leads to only a small improvement in the retrieval quality.

The IWP retrieval using an Artificial Neural Network looks promising, but requires additional work. We can improve the retrieval in various ways. One can make a stronger restriction for homogeneous scenes by looking at MODIS or AVHRR pixels inside the MHS, although this is limited as infrared measurements do not detect the vertical extent of the cloud. Another alternative is to combine MHS with other HIRS channels than those explored so far, or to directly input a combination of MHS and AVHRR for the training. On the other hand, the ANN might be extended to work for more measurements. By having more input parameters or multiple neural networks, the retrieval could work globally,

One can extract additional information from other highresolution data, such as from the Moderate Resolution Imaging Spectroradiometer (MODIS; King and Greenstone, 1999) or the Advanced Very High-Resolution Radiometer (AVHRR; Cracknell, 1997). to better characterise the collocations. Those can be used to make a stronger estimate as to how homogeneous the scene observed by MHS is.

All the applications can be expanded upon and many other applications can be developed.

These and other issues will be adressed in further research. In particular, future work will focus on developing a global IWP product from passive microwave and infrared sensors available on operational polar orbiting satellites.

The collocations are available for public use.

Acknowledgements. The bulk of the work was carried out as part of the Master's Thesis by first author Gerrit Holl. Thanks to the Spacemaster education programme for making this possible. We thank the people, organisations and institutes that have helped us to obtain the satellite data. Thanks to to Lisa Neclos for helping us with archived HIRS data, and to all involved with the NOAA CLASS archive for recent and current MHS, AMSU-B and HIRS measurements. Thanks to CloudSat for making available an accurate IWP product. We thank the UK MetOffice for providing the AAPP package and the ARTS radiative transfer community for its work on ARTS. We would also like to thank the National Graduate School in Space Technology at Luleå University of Technology. Thanks to the OpenStreetMap community for the freely useable map-data in Fig. 1. We would like to thank the AMT Editorial Board, associate editor Bernhard Mayer, and two anonymous reviewers, for their work in improving the article.

Edited by: B. Mayer 


\section{References}

Aoki, T.: A Method for Matching the HIRS/2 and AVHRR Pictures of TIROS-N Satellites, Tech. rep., Meteorological Satellite Center, technical Note No. 2, 1980.

Austin, R. T., Heymsfield, A. J., and Stephens, G. L.: Retrievals of ice cloud microphysical parameters using the CloudSat millimeter-wave radar and temperature, J. Geophys. Res., 114, D00A23, doi:10.1029/2008JD010049, 2009.

Bennartz, R.: Optimal Convolution of AMSU-B to AMSUA, J. Atmos. Oceanic Technol., 17, 1215-1225, doi:10.1175/ 1520-0426(2000)017〈1215:OCOABT $\rangle$ 2.0.CO;2, 2000.

Buehler, S. A. and John, V. O.: A Simple Method to Relate Microwave Radiances to Upper Tropospheric Humidity, J. Geophys. Res., 110, D02110, doi:10.1029/2004JD005111, 2005.

Buehler, S. A., Kuvatov, M., John, V. O., Leiterer, U., and Dier, H.: Comparison of Microwave Satellite Humidity Data and Radiosonde Profiles: A Case Study, J. Geophys. Res., 109, D13103, doi:10.1029/2004JD004605, 2004.

Buehler, S. A., Eriksson, P., Kuhn, T., von Engeln, A., and Verdes, C.: ARTS, the Atmospheric Radiative Transfer Simulator, J. Quant. Spectrosc. Radiat. Transfer, 91, 65-93, doi:10.1016/j. jqsrt.2004.05.051, 2005.

Buehler, S. A., Jiménez, C., Evans, K. F., Eriksson, P., Rydberg, B., Heymsfield, A. J., Stubenrauch, C., Lohmann, U., Emde, C., John, V. O., Sreerekha, T. R., and Davis, C. P.: A concept for a satellite mission to measure cloud ice water path and ice particle size, Q. J. R. Meteorol. Soc., 133, 109-128, doi:10.1002/qj.143, 2007.

Cracknell, A.: The advanced very high resolution radiometer (AVHRR), CRC Press, Boca Raton, FL, United States, 1997.

Davis, C., Emde, C., and Harwood, R.: A 3D Polarized Reversed Monte Carlo Radiative Transfer Model for $\mathrm{mm}$ and submm Passive Remote Sensing in Cloudy Atmospheres, IEEE T. Geosci. Remote, 43, 1096-1101, doi:10.1109/TGRS.2004. 837505, 2005.

Deirmendjian, D.: Complete Microwave Scattering and Extinction Properties of Polydispersed Cloud and Rain Elements, Tech. rep., United States Air Force, RAND, r-422-PR, 1963.

Emde, C., Buehler, S. A., Davis, C., Eriksson, P., Sreerekha, T. R., and Teichmann, C.: A Polarized Discrete Ordinate Scattering Model for Simulations of Limb and Nadir Longwave Measurements in 1D/3D Spherical Atmospheres, J. Geophys. Res., 109, D24207, doi:10.1029/2004JD005140, 2004.

Frey, R. A., Ackerman, S. A., and Soden, B. J.: Climate Parameters from Satellite Spectral Measurements. Part I: Collocated AVHRR and HIRS/2 Observations of Spectral Greenhouse Parameter, J. Climate, 9, 327-344, 1996.

Greenwald, T. J. and Christopher, S. A.: Effect of cold clouds on satellite measurements near $183 \mathrm{GHz}$, J. Geophys. Res., 107, D13, doi:10.1029/2000JD000258, 2002.

Heymsfield, A. J., Protat, A., Austin, R., Bouniol, D., Hogan, R., Delanoë, J., Okamoto, H., Sato, K., van Zadelhoff, G.-J., Donovan, D., and Wang, Z.: Testing IWC Retrieval Methods Using Radar and Ancillary Measurements with In Situ Data, J. Appl. Meteorol. Clim., 47, 135-163, doi:10.1175/2007JAMC1606.1, 2008.

Holz, R. E., Ackerman, S. A., Nagle, F. W., Frey, R., Dutcher, S., Kuehn, R. E., Vaughan, M. A., and Baum, B.: Global Moderate Resolution Imaging Spectroradiometer (MODIS) cloud de- tection and height evaluation using CALIOP, J. Geophys. Res., 113, D00A19, doi:10.1029/2008JD009837, 2008.

Jiménez, C., Eriksson, P., and Murtagh, D.: Inversion of Odin limb sounding submillimeter observations by a neural network technique, Radio Sci., 38, 8062, doi:10.1029/2002RS002644, 2003.

Jiménez, C., Buehler, S. A., Rydberg, B., Eriksson, P., and Evans, K. F.: Performance simulations for a submillimetre wave cloud ice satellite instrument, Q. J. R. Meteorol. Soc., 133, 129-149, doi:10.1002/qj.134, 2007.

John, V. O. and Soden, B. J.: Does convectively-detrained cloud ice enhance water vapor feedback?, Geophys. Res. Lett., 33, L20701, doi:10.1029/2006GL027260, see corrections in John and Soden (2006), GRL, 33, L23701, doi:10.1029/2006GL028663, 2006.

Kahn, B. H., Chahine, M. T., Stephens, G. L., Mace, G. G., Marchand, R. T., Wang, Z., Barnet, C. D., Eldering, A., Holz, R. E., Kuehn, R. E., and Vane, D. G.: Cloud type comparisons of AIRS, CloudSat, and CALIPSO cloud height and amount, Atmos. Chem. Phys., 8, 1231-1248, 2008, http://www.atmos-chem-phys.net/8/1231/2008/.

Kidd, C., Levizzani, V., and Bauer, P.: A review of satellite meteorology and climatology at the start of the twenty first century, Prog. Phys. Geog., 33, 474-489, 2009.

King, M. and Greenstone, R.: EOS reference handbook : a guide to NASA's Earth Science Enterprise and the Earth Observing System, NASA/Goddard Space Flight Center, 1999.

Kleespies, T. J. and Watts, P.: Comparison of simulated radiances, Jacobians and linear error analysis for the Microwave Humidity Sounder and the Advanced Microwave Sounding Unit-B, Q. J. R. Meteorol. Soc., 132, 3001-3010, 2007.

Krasnopolsky, V.: Neural network emulations for complex multidimensional geophysical mappings: Applications of neural network techniques to atmospheric and oceanic satellite retrievals and numerical modeling, Rev. Geophys., 45, RG3009, doi: 10.1029/2006RG000200, 2007.

Labrot, T., Lavanant, L., Whyte, K., Atkinson, N., and Brunel, P.: AAPP Documentation Scientific Description, version 6.0, document NWPSAF-MF-UD-001, Tech. rep., NWP SAF, Satellite Application Facility for Numerical Weather Prediction, 2006.

Liu, G. and Curry, J. A.: Determination of Ice Water Path and Mass Median Particle Size Using Multichannel Microwave Measurements, J. Appl. Meteorol., 39, 1318-1329, 2000.

McFarquhar, G. M. and Heymsfield, A. J.: Parameterization of Tropical Cirrus Ice Crystal Size Distribution and Implications for Radiative Transfer: Results from CEPEX, J. Atmos. Sci., 54, 2187-2200, 1997.

Miller, S. D., Stephen, G. L., Drummond, C. K., Heidinger, A. K., and Partain, P. T.: A multisensor diagnostic satellite cloud property retrieval scheme, J. Geophys. Res., 105, 19955-19971, 2000.

Nagle, F. W.: The Association of Disparate Satellite Observations, in: Second Symposium on Integrated Observing Systems, pp. 49-52, 1998.

Nagle, F. W. and Holz, R. E.: Computationally Efficient Methods of Collocating Satellite, Aircraft, and Ground Observations, J. Atmos. Oceanic Technol., 26, 1585-1595, 2009.

Rogers, R. and Yau, M.: A short course in cloud physics, Pergamon press Oxford, 1979.

Rydberg, B., Eriksson, P., Buehler, S. A., and Murtagh, D. P.: Non- 
Gaussian Bayesian retrieval of tropical upper tropospheric cloud ice and water vapour from Odin-SMR measurements, Atmos. Meas. Tech., 2, 621-637, 2009, http://www.atmos-meas-tech.net/2/621/2009/.

Saunders, R. W., Hewison, T. J., Stringer, S. J., and Atkinson, N. C.: The Radiometric Characterization of AMSU-B, IEEE T. Microw. Theory, 43, 760-771, 1995.

Stephens, G. L.: Cloud feedbacks in the climate system: A critical review, J. Climate, 18, 237-273, 2005.

Stephens, G. L., Vane, D. G., Boain, R. J., Mace, G. G., Sassen, K., Wang, Z., Illingworth, A. J., OConnor, E. J., Rossow, W. B., Durden, S. L., Miller, S. D., Austin, R. T., Benedetti, A., Mitrescu, C., et al.: The Cloudsat Mission and the A-Train, Bull. Amer. Met. Soc., 83, 1771-1790, 2002.

Sun, H., Wolf, W., King, T., Barnet, C., and Goldberg, M.: CoLocation Algorithms for Satellite Observations, in: 86th AMS Annual Meeting, this paper appears in the Proceedings of the 14th Conference on Satellite Meteorology and Oceanography, 2006.

Venema, V., Ament, F., and Simmer, C.: A Stochastic Iterative Amplitude Adjusted Fourier Transform algorithm with improved accuracy, Nonlin. Processes. Geophys., 13, 321-328, 2006.

Waliser, D. E., Li, J.-L. F., Woods, C. P., Austin, R. T., Bacmeister, J., Chern, J., Genio, A. D., Jiang, J. H., Kuang, Z., Meng, H., Minnis, P., Platnick, S., Rossow, W. B., Stephens, G. L., SunMack, S., Tao, W.-K., Tompkins, A. M., Vane, D. G., Walker, C., and $\mathrm{Wu}, \mathrm{D}$.: Cloud ice: A climate model challenge with signs and expectations of progress, J. Geophys. Res., 114, D00A21, doi:10.1029/2008JD010015, 2009.
Weng, F., Zhao, L., Ferraro, R. R., Poe, G., Li, X., and Grody, N. C.: Advanced microwave sounding unit cloud and precipitation algorithms, Radio Sci., 38, 8068, doi:10.1029/2002RS002679, 2003.

Wielicki, B. A. and Parker, L.: On the Determination of Cloud Cover From Satellite Sensors: The Effect of Sensor Spatial Resolution, J. Geophys. Res., 97, 12 799-12 823, 1992.

Wielicki, B. A., Cess, R. D., King, M. D., Randall, D. A., and Harrison, E. F.: Mission to Planet Earth: Role of Clouds and Radiation in Climate, Bull. Amer. Met. Soc., 76, 2125-2153, 1995.

Wu, D. L., Austin, R. T., Deng, M., Durden, S. L., Heymsfield, A. J., Jiang, J. H., Lambert, A., Li, J.-L., Livesey, N. J., McFarquhar, G. M., Pittman, J. V., Stephens, G. L., Tanelli, S., Vane, D. G., and Waliser, D. E.: Comparisons of global cloud ice from MLS, CloudSat, and correlative data sets, J. Geophys. Res., 114, D00A24, doi:10.1029/2008JD009946, 2009.

Zhao, L. and Weng, F.: Retrieval of Ice Cloud Parameters Using the Advanced Microwave Sounding Unit, J. Appl. Meteorol., 41, 384-395, 2002. 